\title{
Dynamics of a forced 2 dof piece-wise linear system by consideration of the weight
}

\author{
A. Ture Savadkoohi ${ }^{1}$, C.-H. Lamarque ${ }^{1}$, and Z. Dimitrijevic ${ }^{2}$ \\ 1 Université de Lyon, ENTPE, DGCB and LTDS, UMR CNRS 5513, rue Maurice Audin, 69518 Vaulx-en-Velin, France \\ 2 PSA Peugeot Citroën Automobiles, DRIA/DSTF/MSMX, route de Gisy, 78943 Vélizy-Villacoublay, France
}

\begin{abstract}
Energy pumping phenomenon between a linear system and a non-smooth system by taking into account the weight of the system is studied. The system faces bifurcation when it reaches to its unstable border and then according to external forcing term it can follow lower stable branch or to face strongly modulated response by hysteresis jumps between its stable branches.
\end{abstract}

\section{Introduction}

It has been proved that a system can be passively controlled by endowing nonlinear innate of coupled cubic systems, namely nonlinear energy sink (NES) to the main one [120]. There has been some attempts to consider the nonsmooth NES systems instead of cubic ones. From them we can mention to the work by Nucera et al. [21] for considering the vibro-impact NES, Gendelman [22] for considering the non-polynomial NES systems and Lamarque et al. [23] for considering piece-wise linear NES. In this paper we try to enlighten the system behavior in terms of the energy transfer between a linear master system and a non-smooth NES considering the weight of the whole system. The multi-scales dynamics of the system at different time scales are detected and are accompanied by numerical evidences. Organization of the paper is as it follows: The system and some analytical treatments are clarified in Sec. 2. The multi-scales dynamics of the system are detected in Sec. 3 while numerical results are collected in Sec. 4. Finally conclusions are given in Sec. 5

\section{Description of the model and some analytical treatments}

Let us consider the system which is preinserted in Fig.1. It is consist of a main structure with the mass $M$ which is weakly coupled to a non-smooth system with the mass $m$. Governing equations of the mentioned academic model under external periodic forcing term $F(t)=\Gamma \sin (\Omega t)$ are as it follows:

$\left\{\begin{array}{l}M \ddot{x}_{1}+k_{1} x_{1}+P\left(x_{1}-x_{2}\right)+\eta\left(\dot{x}_{1}-\dot{x}_{2}\right)+M g=\Gamma \sin (\Omega t) \\ m \ddot{x}_{2}+P\left(x_{2}-x_{1}\right)+\eta\left(\dot{x}_{2}-\dot{x}_{1}\right)+m g=0\end{array}\right.$

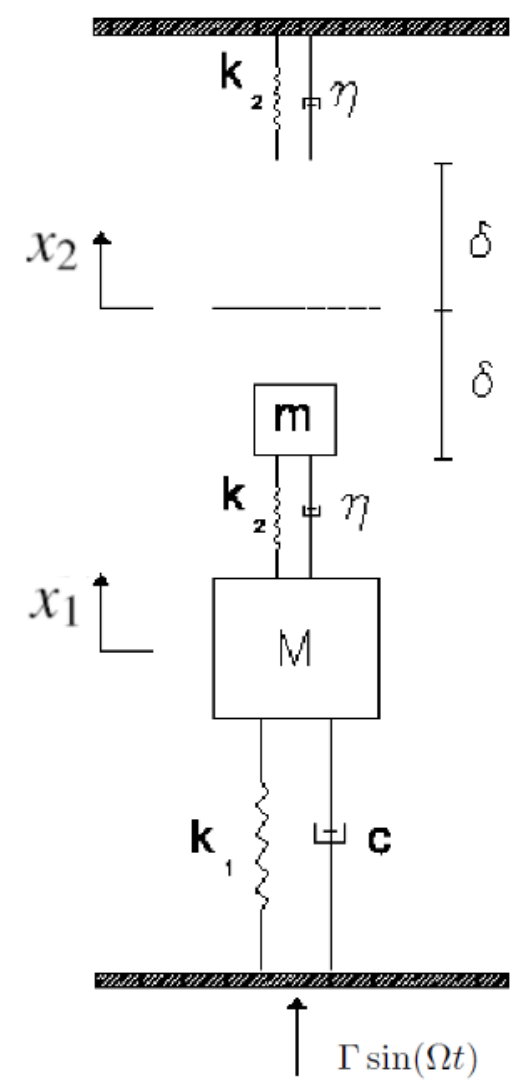

$P$ is the non-smooth potential of the NES which is an odd function and is defined as it follows: 
The system (1) in the domain of the period $T$ of the system $\left(T=t \sqrt{\frac{k_{1}}{M}}=t \vartheta\right)$ can be re-written as:

$$
\left\{\begin{array}{l}
\ddot{y}_{1}+y_{1}+\epsilon \tilde{P}\left(y_{1}-y_{2}\right)+\epsilon \lambda\left(\dot{y}_{1}-\dot{y}_{2}\right)+\gamma=\epsilon f_{0} \sin (\omega T) \\
\epsilon \ddot{y}_{2}+\epsilon \tilde{P}\left(y_{2}-y_{1}\right)+\epsilon \lambda\left(\dot{y}_{2}-\dot{y}_{1}\right)+\epsilon \gamma=0
\end{array}\right.
$$

where $y_{1}$ and $y_{2}$ are shifted displacements $x_{1}$ and $x_{2}$ in domain of $T$ and $\epsilon=\frac{m}{M}, \gamma=\frac{M g}{k_{1}}, \epsilon \lambda=\frac{\eta}{\sqrt{k_{1} M}}, \frac{1}{k_{1}} P(z)=$ $\epsilon \tilde{P}(z), k=\frac{1}{\epsilon} \frac{k_{2}}{k_{1}}, \omega=\frac{\Omega}{\vartheta}$ and $\frac{\Gamma}{k_{1}}=\epsilon f_{0}$. So, the scaled potential of the NES reads as:

$$
\tilde{P}(z)=\left\{\begin{array}{lcc}
0 & \text { if } & -\delta \leq z \leq \delta \\
k(z-\delta) \text { if } & z \geq \delta \\
k(z+\delta) \text { if } & z \leq-\delta
\end{array}\right.
$$

We are interested to study the system (3) in the vicinity of $1: 1$ resonance. Let us transfer the system to the following coordinates:

$$
\left\{\begin{array}{l}
v=y_{1}+\epsilon y_{2} \\
w=y_{1}-y_{2}
\end{array}\right.
$$

Then the system (3) takes following form:

$$
\left\{\begin{array}{l}
\ddot{v}+\frac{1}{1+\epsilon}(v+\epsilon w)+\gamma(1+\epsilon)=\epsilon f_{0} \sin (\omega T) \\
\ddot{w}+\frac{1}{1+\epsilon}(v+\epsilon w)+(1+\epsilon) \tilde{F}(w)+(1+\epsilon) \lambda \dot{w} \\
=\epsilon f_{0} \sin (\omega T)
\end{array}\right.
$$

Let us introduce a modified form of Manevitch's complex variables [24] to the system of (6):

$$
\left\{\begin{array}{l}
B_{1}+\varphi_{1} e^{i \omega T}=\dot{v}+i \omega v \\
B_{2}+\varphi_{2} e^{i \omega T}=\dot{w}+i \omega w
\end{array}\right.
$$

where $B_{1}=i b_{1}, B_{2}=i b_{2}$ and $i=\sqrt{-1}$. We can present the function $\tilde{P}(w)$ in the form of Fourier series:

$$
\begin{aligned}
& \tilde{P}(w)=\tilde{F}\left(\frac{b_{2}}{\omega}-\frac{i}{2 \omega}\left(\varphi_{2} e^{i \omega T}-\varphi_{2}^{*} e^{-i \omega T}\right)\right) \\
& =\sum_{j=-\infty}^{+\infty} f_{j}\left(b_{2}, \varphi_{2}, \varphi_{2}^{*}\right) e^{i \omega j T}
\end{aligned}
$$

where the.$^{*}$ represents the complex conjugate of the function under consideration. The above mentioned variables and assumptions lead us to the zero and first order averaged systems of $(6)(j=0,1$ in Eq. (8)):

$$
\left\{\begin{array}{l}
\frac{1}{1+\epsilon}\left(\frac{b_{1}}{\omega}+\epsilon \frac{b_{2}}{\omega}\right)+(1+\epsilon) \gamma=0 \\
\frac{1}{1+\epsilon}\left(\frac{b_{1}}{\omega}+\epsilon \frac{b_{2}}{\omega}\right)+(1+\epsilon) f_{z}\left(b_{2}, \varphi_{2}, \varphi_{2}^{*}\right)=0
\end{array}\right.
$$

$$
\left\{\begin{array}{l}
\dot{\varphi}_{1}=-\frac{i}{2} \epsilon f_{0}+\frac{i}{2 \omega(1+\epsilon)}\left(\varphi_{1}+\epsilon \varphi_{2}\right)-\frac{i}{2} \omega \varphi_{1} \\
\dot{\varphi}_{2}=-\frac{i}{2} \epsilon f_{0}+\frac{i}{2 \omega(1+\epsilon)}\left(\varphi_{1}+\epsilon \varphi_{2}\right)-\frac{\lambda(1+\epsilon)}{2} \varphi_{2} \\
-\frac{i}{2} \omega \varphi_{2}-(1+\epsilon) f_{f}\left(b_{2}, \varphi_{2}, \varphi_{2}^{*}\right)
\end{array}\right.
$$

$f_{z}\left(b_{2}, \varphi_{2}, \varphi_{2}^{*}\right)$ and $f_{f}\left(b_{2}, \varphi_{2}, \varphi_{2}^{*}\right)$ are zero and first Fourier coefficients which can be evaluated as:

$$
f_{z}\left(b_{2}, \varphi_{2}, \varphi_{2}^{*}\right)=
$$

$$
\begin{aligned}
& \frac{\omega}{2 \pi} \int_{0}^{\frac{2 \pi}{\omega}} \tilde{P}\left(\frac{b_{2}}{\omega}-\frac{i}{2 \omega}\left(\varphi_{2} e^{i \omega T}-\varphi_{2}^{*} e^{-i \omega T}\right)\right) d T \\
& f_{f}\left(b_{2}, \varphi_{2}, \varphi_{2}^{*}\right)= \\
& \frac{\omega}{2 \pi} \int_{0}^{\frac{2 \pi}{\omega}} \tilde{P}\left(\frac{b_{2}}{\omega}-\frac{i}{2 \omega}\left(\varphi_{2} e^{i \omega T}-\varphi_{2}^{*} e^{-i \omega T}\right)\right) e^{-i \omega T} d T
\end{aligned}
$$

It can be proved that:

$$
f_{f}\left(b_{2}, \varphi_{2}, \varphi_{2}^{*}\right)=-\frac{i \varphi_{2}}{2} G_{f}\left(\left|\varphi_{2}\right|^{2}\right)
$$

If $\frac{b_{2}}{\omega}+\frac{\left|\varphi_{2}\right|}{\omega} \leq \delta$

$$
G_{f}\left(\left|\varphi_{2}\right|^{2}\right)=0
$$




$$
f_{z}\left(\left|\varphi_{2}\right|^{2}\right)=0
$$

and if $\frac{b_{2}}{\omega}+\frac{\left|\varphi_{2}\right|}{\omega} \geq \delta$

$$
\begin{aligned}
& G_{f}\left(\left|\varphi_{2}\right|^{2}\right)=\frac{k}{2 \pi \omega}\left(\pi+\frac{2 b_{2}}{\left|\varphi_{2}\right|^{2}} \sqrt{\left|\varphi_{2}\right|^{2}-\left(b_{2}-\delta \omega\right)^{2}}\right. \\
& -\frac{2 \delta \omega}{\left|\varphi_{2}\right|^{2}} \sqrt{\left|\varphi_{2}\right|^{2}-\left(b_{2}-\delta \omega\right)^{2}} \\
& -\frac{2 b_{2}}{\left|\varphi_{2}\right|^{2}} \sqrt{\left|\varphi_{2}\right|^{2}-\left(b_{2}+\delta \omega\right)^{2}} \\
& -\frac{2 \delta \omega}{\left|\varphi_{2}\right|^{2}} \sqrt{\left|\varphi_{2}\right|^{2}-\left(b_{2}+\delta \omega\right)^{2}} \\
& \left.+2 \arccos \left(\frac{b_{2}+\delta \omega}{\left|\varphi_{2}\right|}\right)+2 \arcsin \left(\frac{b_{2}-\delta \omega}{\left|\varphi_{2}\right|}\right)\right)
\end{aligned}
$$

$$
\begin{aligned}
& f_{z}\left(\left|\varphi_{2}\right|^{2}\right)=\frac{k}{\pi \omega}\left(b_{2} \pi+\right. \\
& \sqrt{\left(b_{2}+\left|\varphi_{2}\right|-\delta \omega\right)\left(-b+\left|\varphi_{2}\right|+\delta \omega\right)}- \\
& \sqrt{\left(-b_{2}+\left|\varphi_{2}\right|-\delta \omega\right)\left(b_{2}+\left|\varphi_{2}\right|+\delta \omega\right)} \\
& +\left(b_{2}-\delta \omega\right) \arcsin \left(\frac{b_{2}-\delta \omega}{\left|\varphi_{2}\right|}\right) \\
& \left.+\left(b_{2}+\delta \omega\right) \arcsin \left(\frac{b_{2}+\delta \omega}{\left|\varphi_{2}\right|}\right)\right)
\end{aligned}
$$

In order to reveal the behavior of the averaged systems of (9) and (10) an asymptotic approach [25] by introducing slow times $\tau_{1}, \tau_{2}, \ldots$ with the fast time $\tau_{0}$ can be implemented as follows:

$$
T=\tau_{0}, \quad \tau_{1}=\epsilon \tau_{0}, \cdots,
$$

so,

$$
\frac{d}{d T}=\frac{\partial}{\partial \tau_{0}}+\epsilon \frac{\partial}{\partial \tau_{1}}+\cdots
$$

\section{Multi-scales dynamics of averaged zero and first order systems}

The general form of the system (9) show that $\gamma=f_{z}\left(N_{2}^{2}\right)$. During the $\epsilon^{0}$ order, it leads to:

$$
b_{1}=-\gamma
$$

while during the $\epsilon^{1}$ order we have:

$$
b_{2}=-\gamma(2+\sigma)
$$

Let us assume that $\omega=1+\sigma \epsilon$. The system of (10) at the $\epsilon^{0}$ order yields to:

$$
\begin{gathered}
\frac{\partial \varphi_{1}}{\partial \tau_{0}}=0 \Rightarrow \varphi_{1}=\varphi_{1}\left(\tau_{1}\right) \\
\frac{\partial \varphi_{2}}{\partial \tau_{0}}+\frac{i\left(1-G_{f}\left(\left|\varphi_{2}\right|^{2}\right)\right)+\lambda}{2} \varphi_{2}=\frac{i}{2} \varphi_{1}
\end{gathered}
$$

Considering the system behavior around its fixed points $\left(\frac{\partial \varphi_{2}}{\partial \tau_{0}}=0, \varphi_{2} \rightarrow \Phi\right)$ and $\varphi_{1}=N_{1} e^{i \delta_{1}}$ and $\Phi=N_{2} e^{i \delta_{2}}$, one can reach to following invariant manifold of the system at the $\tau_{0}$ time scale:

$$
N_{1}=N_{2} \sqrt{\lambda^{2}+\left(1-G_{f}\left(N_{2}^{2}\right)\right)^{2}}
$$

It can be proved that the stability border of the above mentioned invariant manifold can be defined as [23]:

$$
\lambda^{2}+\left(1-G_{f}\left(N_{2}^{2}\right)\right)\left(1-H\left(N_{2}^{2}\right) N_{2}-G_{f}\left(N_{2}^{2}\right)\right) \geq 0
$$

where 


$$
H\left(N_{2}^{2}\right)=\left\{\begin{array}{l}
0 \quad \text { if } \quad N_{2}<\delta \\
\frac{2 k}{\pi \omega N_{2}^{3}}\left(b _ { 2 } \left(\sqrt{N_{2}^{2}-\left(b_{2}+a \omega\right)^{2}}-\right.\right. \\
\left.\sqrt{N_{2}^{2}-\left(b_{2}-a \omega\right)^{2}}\right) \\
+a \omega\left(\sqrt{N_{2}^{2}-\left(b_{2}+a \omega\right)^{2}}+\right. \\
\left.\left.\sqrt{N_{2}^{2}-\left(b_{2}-a \omega\right)^{2}}\right)\right) \quad \text { if } \quad N_{2} \geq \delta
\end{array}\right.
$$

An invariant manifold of a system with given parameters and its stable and unstable zones are depicted in Fig. 2.

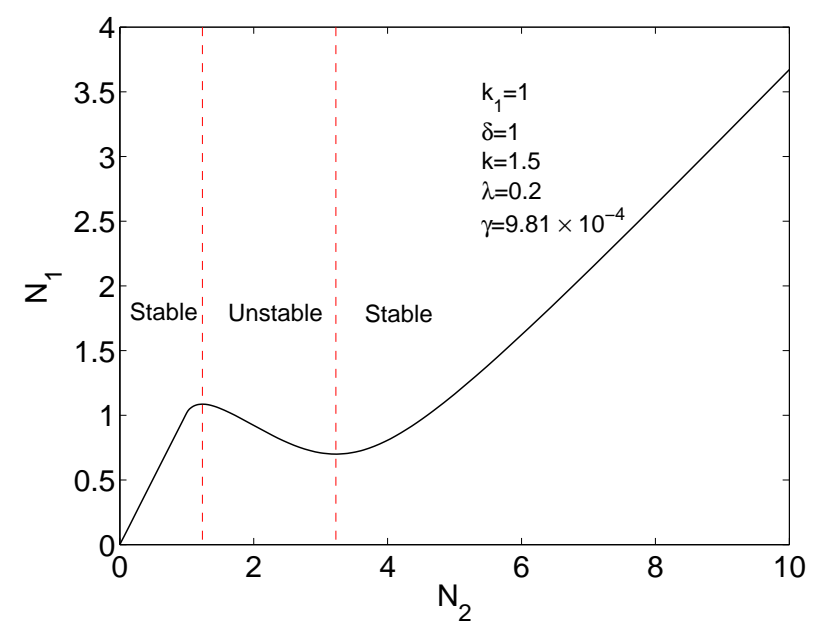

Fig. 2. Invariant manifold of a system and its stable and unstable zones at the $\tau_{0}$ time scale.

At the order of $\epsilon^{1}$ the first equation of the system (10) reads as:

$$
\frac{\partial \varphi_{1}}{\partial \tau_{1}}=-\frac{i}{2} f_{0}+\frac{i}{2}\left(\Phi-\varphi_{1}-\sigma \varphi_{1}\right)-\frac{i}{2} \sigma \varphi_{1}
$$

We would like to detect the behavior of the system at the infinity of $\tau_{1}$ "around" the invariant manifold at the time scale $\tau_{0}$. By considering Eq. (24), the invariant manifold of the system at the $\tau_{1}$ time scale can defined as $\left(\frac{\partial \varphi_{1}}{\partial \tau_{1}}=0\right)$ :

$$
f_{0}=N_{2} \sqrt{\lambda^{2}(1+2 \sigma)^{2}+\left((1+2 \sigma) G_{f}\left(N_{2}^{2}\right)-2 \sigma\right)^{2}}
$$

\section{Numerical results}

The equilibrium state of each mass of the system due to the gravity should be considered in all numerical results $\left(x_{10}\right.$ and $x_{20}$ ). It can be proved that the equilibrium state of the system is defied as:

$$
\left\{\begin{array}{l}
x_{10}=-\frac{M g}{k_{1}}(1+\epsilon)=-\gamma(1+\epsilon) \\
x_{20}=-\frac{M g}{k_{1}}(1+\epsilon)-\delta-\frac{m g}{k_{2}}=-\gamma(1+\epsilon)-\delta-\epsilon \frac{M g}{k_{2}}
\end{array}\right.
$$

This equilibrium points should be considered in all numerical results. We can impose equilibrium states to the numerical results as its follows:

$$
\left\{\begin{array}{l}
v_{\text {numerical }}=\left(y_{1}-x_{10}\right)+\epsilon\left(y_{2}-x_{20}\right) \\
w_{\text {numerical }}=\left(y_{1}-x_{10}\right)-\left(y_{2}-x_{20}\right)
\end{array}\right.
$$

So, $N_{1}$ and $N_{2}$ can be defined in terms of original system of equations ( $N_{1}^{\text {exact }}$ and $\left.N_{2}^{\text {exact }}\right)$ as it follows:

$$
\begin{aligned}
& N_{1}^{\text {exact }}=\sqrt{\left(v_{\text {numerical }}\right)^{2}+\left(\dot{y_{1}}+\epsilon \dot{y_{2}}\right)^{2}} \\
& N_{2}^{\text {exact }}=\sqrt{\left(w_{\text {numerical }}\right)^{2}+\left(\dot{y_{1}}-\dot{y_{2}}\right)^{2}}
\end{aligned}
$$

Let us consider following initial conditions for the system:

$$
\begin{cases}y_{1}(0)=1.5+x_{10}, & \dot{x}_{1}(0)=0 \\ y_{2}(0)=x_{20}, & \dot{x}_{2}(0)=0\end{cases}
$$

Added numerical results to the same system of Fig. 2 for two different amplitudes of external forcing terms are depicted is depicted in Fig. 3 and Fig. 4. When the system reaches to the unstable zone, it tries to reach to other stable zone by a jump between its stable branches through a bifurcation. Then depending on the external forcing term, the system can experience strongly modulated response by hysteresis jumps between its stable branches when it reaches to its fold lines $N_{21}$ and $N_{22}$ (see Fig.4); this behavior leads to beating response of oscillators (see Fig. 5). The reader can refer to [23] for detailed information about bifurcations of the system between its folded singularises. 


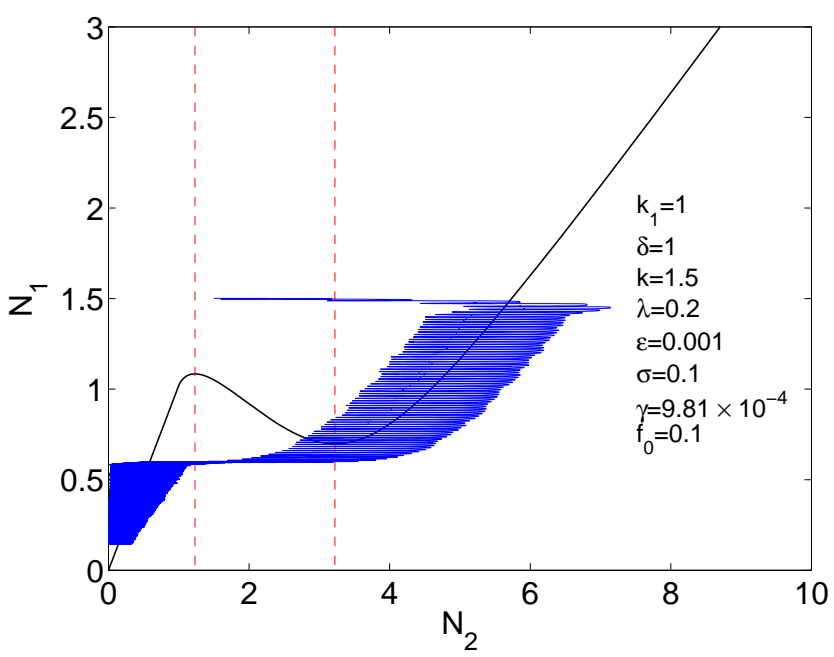

Fig. 3. Invariant manifold of the system in the presence of the gravity and corresponding numerical result: $f_{0}=0.1$

\section{Conclusions}

Multi-scales dynamics of a system with coupled non-smooth NES by taking into account the weight of the system is considered. A modified form of Manevitch's complex variables were considered in zero and first order averaging of the system in order to consider effect of the weight of the system. The system faces bifurcation(s) when it reaches to stability borders. Depending on the external forcing term, the bifurcation can lead the system to very low system amplitudes and/or to strongly modulated response by hysteresis jumps between its stable branches. It is worthwhile to note that other kinds of behaviors can be observed and we did not explain them in this paper and they are beyond the scope of this paper.

\section{References}

1. A. F. Vakakis, Inducing passive nonlinear energy sinks in vibrating systems, ASME Journal of Vibration and

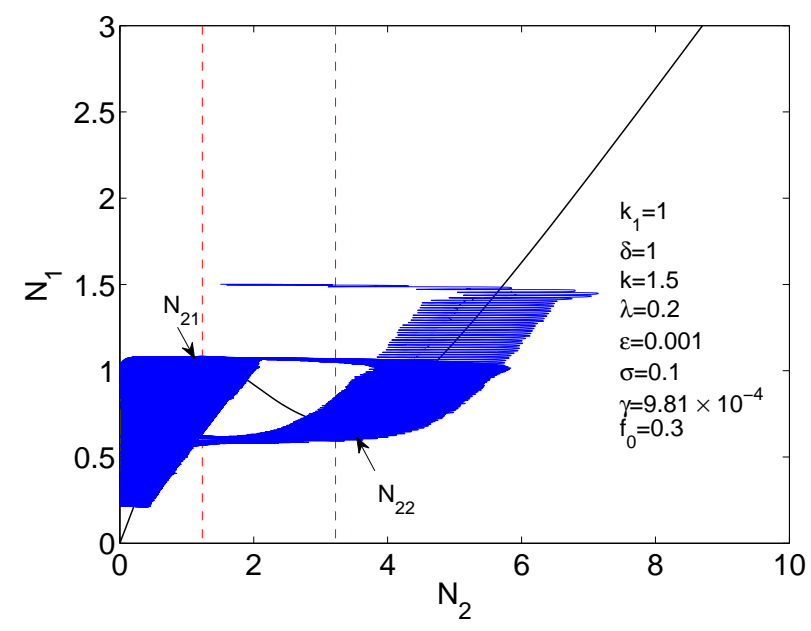

Fig. 4. Invariant manifold of the system in the presence of the gravity and corresponding numerical result: $f_{0}=0.3$

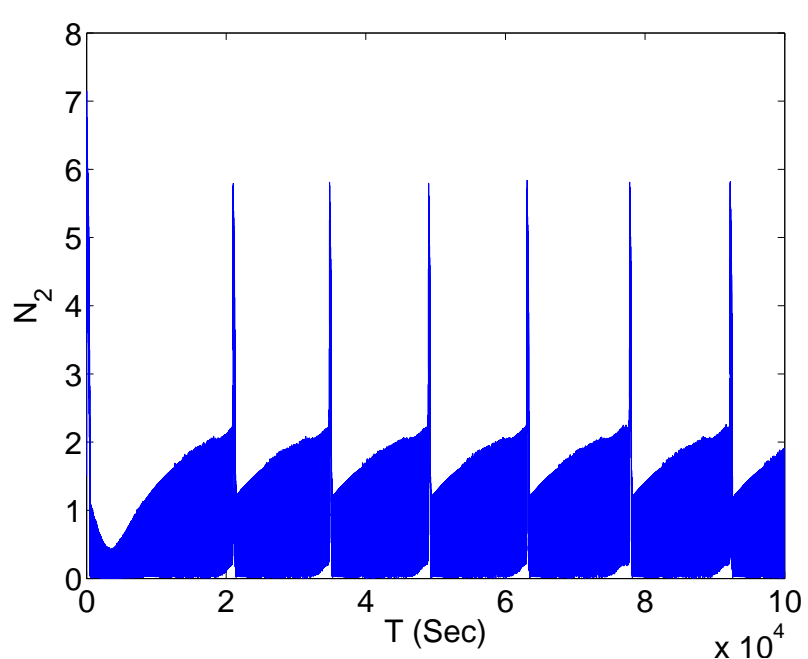

Fig. 5. Beating response of the non-smooth NES: $f_{0}=0.3$

Acoustics, 123, 324-332, 2001.

2. O. V. Gendelman, L. I. Manevitch, A. F. Vakakis, R. M'Closkey, Energy pumping in nonlinear mechanical oscillators I: dynamics of the underlying hamiltonian systems. ASME Journal of Applied Mechanics, 68, 34-41, 2001.

3. A. F. Vakakis, O. V. Gendelman, Energy pumping in nonlinear mechanical oscillators II: resonance capture. ASME Journal of Applied Mechanics, 68, 42-48, 2001.

4. O. V. Gendelman, Bifurcations of nonlinear normal modes of linear oscillator with strongly nonlinear damped attachment. Nonlinear Dynamics, 37, 115-128, 2004.

5. O. V. Gendelman, C.-H. Lamarque, Dynamics of linear oscillator coupled to strongly nonlinear attachment with multiple states of equilibrium. Chaos, Solitons \& Fractals, 24, 501-509, 2005.

6. D. M. McFarland, L. Bergman, A. F. Vakakis, Experimental study of non-linear energy pumping occurring at a single fast frequency. International Journal of NonLinear Mechanics, 40, 891-899, 2005.

7. D. M. McFarland, G. Kerschen, J. J. Kowtko, Y. S. Lee, L. A. Bergman, A. F. Vakakis, Experimental investigation of targeted energy transfers in strongly and nonlinearly coupled oscillators. The Journal of the Acoustical Society of America, 118, 791-799, 2005.

8. O. V. Gendelman, E. Gourdon, C.-H. Lamarque, Quasiperiodic energy pumping in coupled oscillators under periodic forcing. Journal of Sound and Vibration, 294, 651-662, 2006.

9. L. I. Manevitch, E. Gourdon, C.-H. Lamarque, Parameters optimization for energy pumping in strongly nonhomogeneous 2 dof system., Chaos, Solitons \& Fractals, 31, 900-911, 2007.

10. L. I. Manevitch, A. I. Musienko, C.-H. Lamarque, New analytical approach to energy pumping problem in strongly non homogeneous 2 dof systems. Meccanica, 42, 77-83, 2007.

11. L. I. Manevitch, E. Gourdon, C.-H. Lamarque, Towards the design of an optimal energetic sink in a strongly inhomogeneous two-degree-of-freedom system. Journal of Applied Mechanics, 74, 1078-1086, 2007.

12. G. Kerschen, J. J. Kowtko, D. M. McFarland, L. A. Bergman, A. F. Vakakis, Theoretical and experimental 
study of multimodal targeted energy transfer in a system of coupled oscillators. Nonlinear Dynamics, 47, 285309, 2007.

13. G. Kerschen, D. M. McFarland, J. J. Kowtko, Y. S. Lee, L. A. Bergman, A. F. Vakakis, Experimental demonstration of transient resonance capture in a system of two coupled oscillators with essential stiffness nonlinearity. Journal of Sound and Vibration, 299, 822-838, 2007.

14. E. Gourdon, N. A. Alexander, C. A. Taylor, C.-H. Lamarque, S. Pernot, Nonlinear energy pumping under transient forcing with strongly nonlinear coupling: theoretical and experimental results. Journal of Sound and Vibration, 300, 522-551, 2007.

15. E. Gourdon, C.-H. Lamarque, S. Pernot, Contribution to efficiency of irreversible passive energy pumping with a strong nonlinear attachment. Nonlinear Dynamics, 50, 793-808, 2007.

16. A. Ture Savadkoohi, L. I. Manevitch, C.-H. Lamarque, Analysis of the transient behavior in a two dof nonlinear system. Chaos, Solitons \& Fractals, 44 , 450-463, 2011.

17. B. Vaurigaud, L. I. Manevitch, C.-H. Lamarque, Passive control of aeroelastic instability in a long span bridge model prone to coupled flutter using targeted energy transfer. Journal of Sound and Vibration, 330, 25802595, 2011.

18. B. Vaurigaud, A. Ture Savadkoohi, C.-H. Lamarque, Targeted energy transfer with parallel nonlinear energy sinks. Part I: Design theory and numerical results. Nonlinear Dynamics, 66, 763-780, 2011.

19. B. Vaurigaud, A. Ture Savadkoohi, C.-H. Lamarque, Efficient targeted energy transfer with parallel nonlinear energy sinks: theory and experiments. Journal of Computational and Nonlinear Dynamics, 6, 041005-1:04100510, 2011.

20. A. Ture Savadkoohi, B. Vaurigaud, C.-H. Lamarque, S. Pernot, Targeted energy transfer with parallel nonlinear energy sinks, part II: theory and experiments. Nonlinear Dynamics, 67, 37-46, 2012.

21. F. Nucera, A. F. Vakakis, D. M. McFarland, L. A. Bergman, G. Kerschen, Targeted energy transfers in vibro-impact oscillators for seismic mitigation. Nonlinear Dynamics, 50, 651-677, 2007.

22. O. V. Gendelman, Targeted energy transfer in systems with non-polynomial nonlinearity. Journal of Sound and Vibration, 315, 732-745, 2008.

23. C.-H. Lamarque, O. V. Gendelman, A. Ture Savadkoohi, E. Etcheverria, Targeted energy transfer in mechanical systems by means of non-smooth nonlinear energy sink. Acta Mechanica, 221, 175-200, 2011.

24. L. I. Manevitch, The description of localized normal modes in a chain of nonlinear coupled oscillators using complex variables. Nonlinear Dynamics, 25, 95-109, 2001.

25. A. H. Nayfeh, D. T. Mook, Nonlinear Oscillations, John Wiley and Sons, New York, 1979. 